\title{
AN OPEN PROBLEM ON JEŚMANOWICZ' CONJECTURE CONCERNING PRIMITIVE PYTHAGOREAN TRIPLES
}

\author{
Hai YANG AND RUIQIN Fu
}

Xi'an Polytechnic University, P. R. China and Xi'an Shiyou University, P. R.

China

Abstract. Let $m>31$ be an even integer with $\operatorname{gcd}(m, 31)=1$. In this paper, using some elementary methods, we prove that the equation $\left(m^{2}-31^{2}\right)^{x}+(62 m)^{y}=\left(m^{2}+31^{2}\right)^{z}$ has only the positive integer solution $(x, y, z)=(2,2,2)$. This result resolves an open problem raised by $\mathrm{T}$. Miyazaki (Acta Arith. 186 (2018), 1-36) about Jeśmanowicz' conjecture concerning primitive Pythagorean triples.

\section{INTRODUCTION}

Let $\mathbb{Z}, \mathbb{N}$ be the sets of all integers and positive integers, respectively. Let $(a, b, c)$ be a primitive Pythagorean triple with $2 \mid b$. Then we have

$a=m^{2}-n^{2}, b=2 m n, c=m^{2}+n^{2}, m, n \in \mathbb{N}, m>n, \operatorname{gcd}(m, n)=1,2 \mid m n$ and

$$
a^{2}+b^{2}=c^{2} .
$$

In 1956, L. Jeśmanowicz ([2]) conjectured that the equation

$$
a^{x}+b^{y}=c^{z}, x, y, z \in \mathbb{N}
$$

has only the solution $(x, y, z)=(2,2,2)$. Jeśmanowicz' conjecture has been proved to be true in many special cases $([6])$. But, in general, this problem is not solved as yet.

2010 Mathematics Subject Classification. 11D61.

Key words and phrases. Ternary purely exponential Diophantine equation, Jeśmanowicz' conjecture, primitive Pythagorean triple, elementary method.

This work is supported by N.S.F. $(11226038,11371012)$ of P.R. China, the N.S.F. (2017JM1025) of Shaanxi Province, the Education Department Foundation of Shaanxi Province (17JK0323). 
We now consider Jeśmanowicz' conjecture for some fixed $n$. In 1959, W.D. $\mathrm{Lu}([3])$ proved that if $n=1$, then the conjecture is true. After fifty-five years, N. Terai ([7]) solved the case that $n=2$. Very recently, T. Miyazaki ([4]) using Baker's method to prove that, for any fixed $n$ with $n \equiv 3(\bmod 4)$, if $m>C(n)$, where $C(n)$ is an effectively computable constant depending only on $n$, then Jeśmanowicz' conjecture is true. Moreover, he solved the conjecture for some values of $n$ with $n \equiv 3(\bmod 4)$. In the same paper, T. Miyazaki showed that because of the constants $C(n)$ obtained from Baker's method are so large, Jeśmanowicz' conjecture is not settled for several small values of $n$ with $n \equiv 3(\bmod 4)$. The smallest one is $n=31$. Thus, he raised the following as an open problem.

Problem. Prove Jeśmanowicz' conjecture for $n=31$.

TheOREM 1.1. Let $m>31$ be an even integer with $\operatorname{gcd}(m, 31)=1$, the equation

$$
\left(m^{2}-31^{2}\right)^{x}+(62 m)^{y}=\left(m^{2}+31^{2}\right)^{z}, x, y, z \in \mathbb{N}
$$

has only the solution $(x, y, z)=(2,2,2)$.

\section{Preliminaries}

Lemma 2.1 ([5, Section 15.2]). For any positive integer $\ell$, every solution $(X, Y, Z)$ of the equation

$$
X^{2}+Y^{2}=Z^{\ell}, X, Y, Z \in \mathbb{N}, \operatorname{gcd}(X, Y)=1,2 \mid Y
$$

can be expressed as

$$
\begin{gathered}
X+Y \sqrt{-1}=\lambda_{1}\left(f+\lambda_{2} g \sqrt{-1}\right)^{\ell}, \lambda_{1}, \lambda_{2} \in\{-1,1\}, \\
Z=f^{2}+g^{2}, f, g \in \mathbb{N}, \operatorname{gcd}(f, g)=1,2 \mid f g .
\end{gathered}
$$

LEMMA 2.2. Let $p$ be an odd prime, and let $f, g, \ell$ be positive integers such that $\operatorname{gcd}(f, g)=1, p \mid g$ and $2 \nmid \ell$. If $p^{e} \| \ell$, where $e$ is a nonnegative integer, then

$$
p^{e} \| \sum_{i=0}^{(\ell-1) / 2}\left(\begin{array}{c}
\ell \\
2 i+1
\end{array}\right) f^{\ell-2 i-1}\left(-g^{2}\right)^{i} .
$$

Proof. Since $\operatorname{gcd}(f, g)=1$ and $p \mid g$, we have $p \nmid f$. Hence, if $e=0$, then $p \nmid \ell$,

$$
\sum_{i=0}^{(\ell-1) / 2}\left(\begin{array}{c}
\ell \\
2 i+1
\end{array}\right) f^{\ell-2 i-1}\left(-g^{2}\right)^{i} \equiv \ell f^{\ell-1} \not \equiv 0 \quad(\bmod p)
$$

and (2.1) is true.

If $e>0$, then

$$
p^{e} \| \ell f^{l-1} .
$$


For any positive integer $i$, let $p^{s_{i}} \| 2 i+1$. Since $p^{s_{i}} \leq 2 i+1$, we have

$$
s_{i} \leq \frac{\log (2 i+1)}{\log p} \leq \frac{\log (2 i+1)}{\log 3}<2 i .
$$

Hence, by (2.3), we get

$$
\begin{aligned}
\left(\begin{array}{c}
\ell \\
2 i+1
\end{array}\right) f^{\ell-2 i-1}\left(-g^{2}\right)^{i} & \equiv(-1)^{i} \ell\left(\begin{array}{c}
\ell-1 \\
2 i
\end{array}\right) f^{\ell-2 i-1} \frac{g^{2 i}}{2 i+1} \\
& \equiv 0 \quad\left(\bmod p^{e+1}\right), i=1, \cdots, \frac{\ell-1}{2} .
\end{aligned}
$$

Therefore, by (2.2) and (2.4), we obtain (2.1). The lemma is proved.

Let $\alpha, \beta$ be algebraic integers. If $\alpha+\beta$ and $\alpha \beta$ are nonzero coprime integers and $\alpha / \beta$ is not a root of unity, then $(\alpha, \beta)$ is called a Lucas pair. Let $A=\alpha+\beta$ and $B=\alpha \beta$. Then we have

$$
\alpha=\frac{1}{2}(A+\lambda \sqrt{D}), \beta=\frac{1}{2}(A-\lambda \sqrt{D}), \lambda \in\{-1,1\},
$$

where $D=A^{2}-4 B$. Further, for any nonnegative integer $j$, one defines the corresponding sequence of Lucas numbers by

$$
L_{j}(\alpha, \beta)=\frac{\alpha^{j}-\beta^{j}}{\alpha-\beta} .
$$

Obviously, $L_{j}(\alpha, \beta)(j=1,2, \cdots)$ are nonzero integers.

Lemma 2.3 ([1, Theorems IV and XII $])$. Let $p$ be an odd prime such that $p \nmid A B D$ and

$$
p \mid L_{r}(\alpha, \beta)
$$

for some positive integer $r$. Further, let $r_{1}$ be the least value of $r$ with (2.6). Then we have

(i) A positive integer $r$ satisfies (2.6) if and only if $r_{1} \mid r$.

(ii) $p-(D / p) \equiv 0\left(\bmod r_{1}\right)$, where $(* / *)$ is the Legendre symbol.

LEMma 2.4. For any real number $t$ with $t \geq 9$, we have

$$
0.2180 t+\frac{1}{2} \log 1488>\log t .
$$

Proof. Let $f(t)=0.2180 t+\frac{1}{2} \log 1488-\log t$. Since $f^{\prime}(t)=0.2180-1 / t$, where $f^{\prime}(t)$ is the derivative of $f(t)$, we have $f^{\prime}(t)>0$ for $t \geq 9$. Therefore, if $t \geq 9$, then $f(t) \geq f(9)=0.2180 \times 9+\frac{1}{2} \log 1488-\log 9>3.4173>0$. Thus the lemma is proved. 


\section{Proof of Theorem 1.1}

In this section, we assume that $(x, y, z)$ is a solution of (1.2) with $(x, y, z) \neq(2,2,2)$. By [4], it suffices to consider the case that $x, y, z$ and $m$ satisfy

$$
\begin{gathered}
x \equiv y \equiv 2 \quad(\bmod 4), 2 \nmid z, \\
x<z
\end{gathered}
$$

and

$$
2^{3} \| m .
$$

Lemma 3.1. $y>z>y / 2, y \geq 6$ and $z>3$.

Proof. Since $x<z$ by (3.2), if $y \leq z$, then from (1.1) and (1.2) we get $\left(m^{2}-31^{2}\right)^{z}+(62 m)^{z}>\left(m^{2}-31^{2}\right)^{x}+(62 m)^{y}=\left(m^{2}+31^{2}\right)^{z}=\left(\left(m^{2}-\right.\right.$ $\left.31)^{2}+(62 m)^{2}\right)^{z / 2}>\left(m^{2}-31^{2}\right)^{z}+(62 m)^{z}$, a contradiction. So we have $y>z$. On the other hand, since $62 m>\left(m^{2}+31^{2}\right)^{1 / 2}$, by (1.2), we get $\left(m^{2}+31^{2}\right)^{z}>(62 m)^{y}>\left(m^{2}+31^{2}\right)^{y / 2}$ and $z>y / 2$.

Since $(x, y, z) \neq(2,2,2)$ and $x \equiv y \equiv 2(\bmod 4)$ by (3.1), we have $\max \{x, y\}>2, z>2$ and $y \geq 6$. Further, since $z>y / 2$, we get $z>3$. The lemma is proved.

LEMma 3.2. $3 \mid m$.

Proof. If $3 \nmid m$, then $m^{2}-31^{2} \equiv 1-1 \equiv 0(\bmod 3)$ and $m^{2}+31^{2} \equiv 1+$ $1 \equiv 2(\bmod 3)$. Since $2 \mid m$ and $2 \nmid z$, by $(1.2)$, we get $1=\left(\left(m^{2}+31^{2}\right)^{z} / 3\right)=$ $\left(\left(m^{2}+31^{2}\right) / 3\right)=(2 / 3)=-1$, a contradiction. Thus, the lemma is proved.

Since $2 \nmid z$ and $2 \mid m$, applying Lemma 2.1 to (1.2), we have

$$
\begin{aligned}
& \left(m^{2}-31^{2}\right)^{x / 2}=f\left|\sum_{i=0}^{(z-1) / 2}\left(\begin{array}{c}
z \\
2 i
\end{array}\right) f^{z-2 i-1}\left(-g^{2}\right)^{i}\right|, \\
& (62 m)^{y / 2}=g\left|\sum_{i=0}^{(z-1) / 2}\left(\begin{array}{c}
z \\
2 i+1
\end{array}\right) f^{z-2 i-1}\left(-g^{2}\right)^{i}\right|, \\
& m^{2}+31^{2}=f^{2}+g^{2}, f, g \in \mathbb{N}, \operatorname{gcd}(f, g)=1,2 \nmid f, 2 \mid g .
\end{aligned}
$$

By (3.3) and (3.5), we get

$$
2^{2 y} \mid g .
$$

Lemma 3.3. $3 \mid g$ and $31 \mid g$. 
Proof. By Lemma 3.2, we have $3 \mid m$. Hence $3 \nmid m^{2}-31^{2}$, and by (3.4), we get $3 \nmid f$. If $3 \nmid g$, then from (3.5) we obtain

$$
\begin{aligned}
0 & \equiv \sum_{i=0}^{(z-1) / 2}\left(\begin{array}{c}
z \\
2 i+1
\end{array}\right) f^{z-2 i-1}\left(-g^{2}\right)^{i} \equiv \sum_{i=0}^{(z-1) / 2}(-1)^{i}\left(\begin{array}{c}
z \\
2 i+1
\end{array}\right) \\
& \equiv \pm 2^{(z-1) / 2} \not \equiv 0 \quad(\bmod 3),
\end{aligned}
$$

a contradiction. So we have $3 \mid g$.

Let

$$
\alpha=f+g \sqrt{-1}, \beta=f-g \sqrt{-1} .
$$

Notice that $\alpha+\beta=2 f, \alpha \beta=f^{2}+g^{2},(\alpha+\beta)^{2}-4 \alpha \beta=-4 g^{2}, \operatorname{gcd}(f, g)=$ $\operatorname{gcd}\left(2 f g, f^{2}+g^{2}\right)=1$ and $\alpha / \beta=\left(\left(f^{2}-g^{2}\right)+2 f g \sqrt{-1}\right) /\left(f^{2}+g^{2}\right)$ is not a root of unity. Then $(\alpha, \beta)$ is a Lucas pair. Further, let $L_{j}(\alpha, \beta)(j=0,1, \cdots)$ be the corresponing sequence of Lucas numbers defined as in (2.5). By (2.5), (3.5) and (3.8), we have

$$
(62 m)^{y / 2}=g\left|L_{z}(\alpha, \beta)\right| .
$$

If $31 \nmid g$, then from (3.9) we get

$$
31 \mid L_{z}(\alpha, \beta) .
$$

We see from (3.10) that

$$
31 \mid L_{r}(\alpha, \beta)
$$

for some positive integers $r$. Let $r_{1}$ be the least value of $r$ with (3.11). Since $f^{2}+g^{2}=m^{2}+31^{2}$ and $31 \nmid m$, we have $31 \nmid f g\left(f^{2}+g^{2}\right)$. Hence by $(i)$ of Lemma 2.3, we see from (3.10) that

$$
r_{1} \mid z \text {. }
$$

On the other hand, since $\left(-4 g^{2} / 31\right)=(-1 / 31)=-1$, by $(i i)$ of Lemma 2.3, we have

$$
31+1 \equiv 2^{5} \equiv 0 \quad\left(\bmod r_{1}\right) .
$$

Further, since $L_{1}(\alpha, \beta)=1$ and $31 \mid L_{r_{1}}(\alpha, \beta)$, we have $r_{1}>1$. Therefore, we find from (3.13) that $2 \mid r_{1}$. But, since $2 \nmid z,(3.12)$ is false. Thus, we get $31 \mid g$. The lemma is proved.

Lemma 3.4. $m>g$.

Proof. By assumption $31 \nmid m, 31 \mid g$ of (3.3) and (3.7), we have $m \neq g$. Since $m \equiv g \equiv 0(\bmod 24)$ by Lemmas 3.2 and 3.3 , if $m<g$, then we have $g \geq m+24$. Hence, by (3.6), we get $m^{2}+31^{2}=f^{2}+g^{2} \geq 1+(m+24)^{2}=$ $m^{2}+48 m+577$, whence we obtain $16 \geq m \geq 31$, a contradiction. So we have $m>g$. The lemma is proved.

Lemma 3.5 ((i) of Lemma 8.1 in [1]). $z-x>(\log m) / \log 31$. 
Let

$$
3^{e_{1}}\left\|z, 31^{e_{2}}\right\| z, e_{1}, e_{2} \in \mathbb{Z}, e_{1} \geq 0, e_{2} \geq 0 .
$$

By Lemmas 2.2 and 3.3, we have

$$
3^{e_{1}}\left\|\sum_{i=0}^{(z-1) / 2}\left(\begin{array}{c}
z \\
2 i+1
\end{array}\right) f^{z-2 i-1}\left(-g^{2}\right)^{i}, 31^{e_{2}}\right\| \sum_{i=0}^{(z-1) / 2}\left(\begin{array}{c}
z \\
2 i+1
\end{array}\right) f^{z-2 i-1}\left(-g^{2}\right)^{i} .
$$

Hence, by (3.5) and (3.15), we get

$$
3^{y / 2-e_{1}}\left|g, \quad 31^{y / 2-e_{2}}\right| g .
$$

Further, by (3.7) and (3.16), we obtain

$$
g \geq \frac{1488^{y / 2}}{3^{e_{1}} 31^{e_{2}}} .
$$

Therefore, by Lemma 3.4, we get from (3.17) that

$$
\log m>\log g \geq \frac{y}{2} \log 1488-\left(e_{1} \log 3+e_{2} \log 31\right) .
$$

By Lemmas 3.1 and 3.5, if $\left(e_{1}, e_{2}\right)=(0,0)$, then from $(3.18)$ we get

$$
\begin{aligned}
\log m & \geq \frac{y}{2} \log 1488>\frac{z}{2} \log 1488>\frac{1}{2}(z-x) \log 1488 \\
& >\frac{(\log m)(\log 1488)}{2 \log 31}>\log m
\end{aligned}
$$

a contradiction.

If $\left(e_{1}, e_{2}\right) \neq(0,0)$, then either $3 \mid z$ or $31 \mid z$. Since $z>3$ by Lemma 3.1, we have $z \geq 9$. By (3.14), we have $3^{e_{1}} 31^{e_{2}} \mid z$. It implies that

$$
e_{1} \log 3+e_{2} \log 31 \leq \log z \text {. }
$$

Hence, since $y>z$ and $y \geq z+1$, by (3.18), we get

$$
\log m \geq \frac{z}{2} \log 1488-\left(\log z-\frac{1}{2} \log 1488\right) .
$$

Recall that $z \geq 9$, by Lemma 2.4 , we have $\log z-\frac{1}{2} \log 1488<0.2180 z$. Therefore, by (3.19), we get

$$
\begin{aligned}
\log m & >\left(\frac{1}{2} \log 1488-0.2180\right) z>3.4345 z>3.4345(z-x) \\
& >\frac{3.4345 \log m}{\log 31}>\log m,
\end{aligned}
$$

a contradiction.

To sum up, the theorem is proved. 


\section{REFERENCES}

[1] R. D. Carmichael, On the numerical factors of arithmetic forms $\alpha^{n} \pm \beta^{n}$, Ann. of Math. (2) 15 (1913/1914), 49-70.

[2] L. Jeśmanowicz, Several remarks on Pythagorean numbers, Wiadom. Mat. (2) 1 (1955/1956), 196-202.

[3] W. D. Lu, On Pythagorean numbers $4 n^{2}-1,4 n$ and $4 n^{2}+1$, J. Sichuan Univ. Nat. Sci. 5 (1959), 39-42.

[4] T. Miyazaki, Contributions to some conjectures on a ternary exponential Diophantine equation, Acta Arith. 186 (2018), 1-36.

[5] L. J. Mordell, Diophantine equations, Academic Press, London-New York, 1969.

[6] G. Soydan, M. Demirci, I. N. Cangül and A. Togbé, On the conjecture of Jeśmanowicz, Int. J. Appl. Math. Stat. 56 (2017), 46-72.

[7] N. Terai, On Jeśmanowicz' conjecture concerning primitive Pythagorean triples, J. Number Theory. 141 (2014), 316-323.

H. Yang

School of Science

Xi'an Polytechnic University

Xi'an, Shaanxi, 710048

P.R. China

E-mail: xpuyhai@163.com

R. Fu

School of Science

Xi'an Shiyou University

Xi'an, Shaanxi, 710065

P.R. China

E-mail: xsyfrq@163.com

Received: 16.4.2019.

Revised: 31.5.2019. 